\title{
BIBLIOGRAPHIE \\ des oeuvres du prof. Pavao Tekavčić
}

\section{A. Livres}

1. Uvod u vulgarni latinitet (s izborom tekstova), Sveučilište u Zagrebu, Zagreb 1970.

2. Grammatica storica dell'italiano (I Fonematica, II Morfosintassi, III Lessico), il Mulino, Bologna, $1972^{1}, 1980^{2}$.

3. Uvod u lingvistiku za studente talijanskog jezika i književnosti, Sveučilišna naklada Liber, Zagreb 1979.

B. Monographies, études, articles

1958:

4. Terminologia viticola e vinicola nel dialetto istrioto di Dignano, «Studia Romanica et Anglica Zagrabiensia» (SRAZ) 5, pgs. 67-76.

1959:

5. Due voci romene in un dialetto serbocroato dell'isola di Veglia (Krk), «SRAZ» 7, pgs. 35-38.

1960:

6. Un testo istrioto, «SRAZ» $9-10$, pgs. $75-84$.

1961:

7. Toponomastica romanza di Dignano d'Istria, «SRAZ» 11, pgs. 67-90.

8. Les groupes consonantiques dans l'istroroman de Dignano, Godišnjak Naučnog društva NRBiH II, Sarajevo, pgs. 129-138.

1963:

9. Un problema della fonematica italiana: la variazione s/ts, «SRAZ» $15-16$, pgs. 99-114.

1966:

10. Un tentativo di classificazione strutturale del verbi "irregolari" nell'istroromanzo di Dignano, «SRAZ» 21-22, pgs. 39-56. 
1967:

11. Il non-accordo tra il predicato composto con 'essere' ed il soggetto invertito nei dialetti istroromanzi, «SRAZ» 23, pgs. 161-194.

12. Sulla motivazione nella formazione delle parole, ib., pgs. 87-102.

13. Današnji istroromanski dijalekt Vodnjana, «Rad» JAZU 348, pgs. 141-288.

14. Ligure antico peizem, «Lingua Nostra» $28 / 2$, pgs. 56-58.

15. Quelques problèmes de l'interprétation structurale des formes verbales istroromanes, «Revue Roumaine de Linguistique» (RRL) 12/3, pgs. 209-228.

16. Su alcune particolarità nella coniugazione rovignese, «Travaux de Linguistique et de Littérature» (TraLiLi) 5/1, Strasbourg, pgs. 225-233.

1968:

17. Sur le superlatif italien et roman, «SRAZ» $25-26$, pgs. $23-42$.

18. Sull'analisi morfematica di un tipo di derivati italiani (il problema dei cosiddetti interfissi), ib., pgs. 69-85.

19. Il fattore morfematico nelle grafie ho, hai, ha, hanno, ib., pgs. 103-108.

20. Formazione delle parole nell' istroromanzo dignanese, «Lingua e Stile» (LeS) $3 / 2$, pgs. 125-180.

21. Otzv. apsolutnom superlativu u modernom talijanskom jeziku, «Živi jezici» 9/1-4, pgs. $15-20$.

1969:

22. Testi istroromanzi dignanesi, «TraLiLi» 7/1, pgs. 275-303.

1970:

23. Saggio di un'analisi del sistema verbale italiano, «LeS» 5/1, pgs. 1-23.

24. Concetti negativi nella formazione delle parole dell'italiano d'oggi, «Beiträge zur romanischen Philologie» 9/2, Berlin, pgs. 279-303.

25. Iz povijesti istroromanskih govora, «Filologija» 6, pgs. 283-299.

26. Caratteristiche del sistema fonematico dignanese, Actele celui de-al XII-lea Congres Internaţional de Lingvistică şi Filologie Romanică I, Bucureşti, pgs. 419-423.

27. Sulla molteplicità dei riflessi delle vocali latine nei dialetti istroromanzi, «RRL» 15/3, pgs. 223-240.

1971:

28. Attrazione formale e contatto semantico, «SRAZ» 29-32, pgs. 55-64.

29. Intorno al dignanese zon, zoyn 'andiamo', ib., pgs. 121-130.

30. Problematika istroromanskih studija, «Dometi», Rijeka, pgs. 56-62.

31. Jedna moguća rumunjska etimologija u hrvatskosrpskom jeziku, Radovi I Simpozijuma o srpsko(jugoslovensko)-rumunskim odnosima, Pančevo, pgs. 409-416.

32. Il dignanese di Ive ed il dignanese di oggi, «RRL» 16/3, pgs. 215-240. 
1972:

33. Sull'alternanza morfematica nel verbo italiano, «Linguistica» 12 , Ljubljana, pgs. 269-300.

34a. Sequenze omofone nella formazione delle parole (I), «Suvremena lingvistika» (SL) 5-6, Zagreb, pgs. 27-36.

1973:

34b. Sequenze omofone nella formazione delle parole (II), «SL» 7-8, pgs. 29-39.

35. Il comune e lo specifico nel dominio istroromanzo, «SRAZ»33-36, pgs. 639-678.

36. Un tipo di alterati nell'istroromanzo dignanese (intorno all'etimologia del dignanese sión 'uccello'), «Revue de Linguistique Romane» (RLiR) 37, pgs. 387-397.

37. Sul vocalismo neolatino autoctono nelle coste orientali dell'Adriatico, «Bollettino dell'Atlante Linguistico Mediterraneo» (BALM) 13-15, pgs. 57-92.

38a. Abbozzo del sistema morfosintattico del soprasilvano odierno (I), «SRAZ» 33-36, pgs. 359-488.

1974:

38b. Abbozzo del sistema morfosintattico del soprasilvano odierno (II), «SRAZ» 37, pgs. 5-134.

39. Lingvistički aspekti vodnjanskih nadimaka, «Onomastica Jugoslavica» (On Jug) 3-4, pgs. $161-177$.

1975:

40. Caratteristiche e problemi del verbo istroromanzo, «SRAZ» 39, pgs. 55-105.

41. Agli albori dell'italiano, «Linguistica» 15 , pgs. 209-239.

42. Sugli aggettivi italiani tipo cuneiforme, imberbe, ventenne, «Studi di Grammatica Italiana» 4 , pgs. $71-84$.

1976:

43. Interferenze linguistiche istroromanzo-venete: sulle vocali finali nell'istroromanzo, Atti del XIV Congresso Internazionale di Linguistica e Filologia Romanica II, Napoli, pgs. 447-467.

44. O kriterijima stratifikacije $i$ regionalne diferencijacije jugoslavenskog romanstva u svjetlu toponomastike, «OnJug» 6, pgs. 35-56.

45. Alcune riflessioni a proposito delle formazioni scientifiche moderne con elementi greci e/o latini, «SL»13-14, pgs. 11-18.

46. L'opera di Friedrich Schürr alla luce dei suoi recenti contributi linguistici e letterari, «SRAZ» 41-42, pgs. 129-165.

47. Per un atlante linguistico istriano (con speciale riguardo ai dialetti istroromanzi), ib., pgs. 227-240. 
1977:

48. Sulla forma verbale vegliota féro e sull'origine del futuro veglioto, «Incontri Linguistici» (IncLing) 3/1, pgs. 71-89.

49. O problematici tvorbe riječi, «Strani jezici» (StrJez), Zagreb, 3-4, pgs. $139-148$.

50. Historijske komponente istroromanske sintakse, en: Problemi polskiej skŁadni historycznej (Konferencja Naukowa, Mogilany 1976), Kraków, pgs. 102-126.

51. Problemi di ricostruzione nel verbo istroromanzo, en: SLI 11: Problemi della ricostruzione in linguistica, Roma, pgs. 177-188.

52. Problemi teorici e metodologici nella ricostruzione dell'istroromanzo, «SRAZ» 43, pgs. 35-54.

53. La problematica del futuro nel retoromanzo svizzero (a proposito di una recente pubblicazione), ib., pgs, 139-155.

1978:

54. Aspetti di formazione delle parole ne "La lingua delle Pagine Gialle", «SRAZ» XXIII/1-2, pgs. 435-446.

55. Sintassi e semantica nella coordinazione avversativa e sostitutiva, "Linguistica) 18 , pgs. $237-258$.

56. Istroromansko zor, hrvatsko i slovensko žor, žur itd. 'surutka', «Filologija» 8 , pgs. $333-340$.

57. L'indigeno e l'alloglotto nell'ordine delle parole di un idioma di frontiera: l'istroromanzo, en: Wortstellung und Bedeutung I, Tübingen, pgs. $67-77$.

1979:

58. Il posto dell'istroromanzo nella Romània Circumadriatica, «SRAZ» XXIV/1-2, pgs. $21-46$.

59. L'épigraphie latine et le problème de la différenciation du latin (à propos de récentes contributions américaines), «Arheološki Vestnik» (ArVest), Ljubljana, 30, pgs. 559-576.

60. La morfosintassi nell'individualità dell'istroromanzo, «Linguistica» 19, pgs.143-156.

61. (en collaboration avec Željka Rančigaj) Tanto iniziale di frase e parole affini, considerazioni semantico-sintattiche, ib., pgs. 199-222.

1980:

62. O nekim "posebnim" tipovima rečenica, prilog problemu klasifikacije, «StrJez»1, pgs. 2-13.

63. Tvorba riječi $u$ našim suvremenim udžbenicima talijanskog jezika, «StrJez» 4, pgs. 290-302.

64. Pluralità di fattori e poligenesi dell'evoluzione linguistica, Zbornik Pedagoškog fakulteta u Rijeci 2, pgs. 247-258. 
1981:

65. É proprio cosi chiaro come sembra?, «Scuola Nostra»12, Rijeka, pgs. 5-16.

66. Rad na jezičnom odgoju i kulturi govora u današnjoj Italiji, «StrJez» 3, pgs. 167-180.

67. Costanza, ripetizione, regressione, unicità nell'evoluzione dell'italiano letterario, «Linguistica» 21 , pgs. $251-275$.

68. Il soprasilvano. Ritratto linguistico della maggiore delle varietà romance, «Ladinia» 5, pgs. 271-291.

69. Romanistična premišljanja in opazke o izgovarjavi klasične latinščine, «ArVest» 32 , pgs. $625-630$.

1982:

70. Intorno ad alcuni ribelli nella tipologia, «Studije iz kontrastivne analize italijanskog i srpskohrvatskog jezika» Beograd, 2, pgs. 5-22.

71. Giuseppe Moscheni "Bara Bepi Belisa" nei ricordi, «La battana» 63-64, Rijeka, pgs. 165-169.

72. O prilozima $i$ "prilozima", sintakto-semantičke, pragmalingvističke $i$ kontrastivne bilješke, «StrJez» 1-2, pgs. 3-12.

73. Motovun i Flaveyco, «OnJug» 9, pgs. 129-135.

74. L'importanza e l'interesse degli studi istroromanzi per la linguistica romanza e generale, «RLiR» 46, pgs. 271-298.

75. Indirizzi linguistici attuali nel dominio istroromanzo, «Linguistica» 22, pgs. 91- 125 .

76. Definicije $i$ klasifikacije rečenice $i$ perioda $u$ našim suvremenim udžbenicima talijanskog jezika, «StrJez» 3-4, pgs. 121-136.

77. Per un insegnamento moderno della grammatica, Zbornik Pedagoškog fakulteta u Rijeci 4, pgs. 231-240.

78. Le due sponde dell'Adriatico nei due millenni di storia linguistica romanza, «Abruzzo», Roma, 20/numm.1-3, pgs. 41-60.

1983:

79. Riflessioni su alcuni aspetti sociolinguistici dello studio del latino volgare, «IncLing» 7, str. 81-96.

80. Fonetica e semantica nell'alternanza verbale italiana (a proposito dell'evoluzione EXIRE > uscire), en: Scritti linguistici in onore di Giovan Battista Pellegrini II, Pisa, pgs. 1189-1200.

81. Problemi di grafia e di trascrizione nei testi istroromanzi, Radovi Pedagoškog fakulteta u Rijeci, OOUR nastavne djelatnosti Pula 4, pgs. 135-149.

82. Osservazioni sulla lingua dei testi istroromanzi contemporanei, en: Linguistica e Dialettologia Veneta, Studi offerti a Manlio Cortelazzo dai colleghi stranieri, Tübingen, pgs. 101-111. 
83. Le espressioni per il 'vincolo' nell'istroromanzo attuale, en: Romanica Gandensia XX: Verbe et phrase dans les langues romanes, Mélanges offerts à Louis Mourin, Gent, pgs. 221-230.

84. O hrvatskom ili srpskom participu na -vši i njegovim talijanskim ekvivalentima, Zbornik Pedagoškog fakulteta u Rijeci 5, pgs. 201-208.

85. L'alternanza morfematica nell'istroromanzo. Tentativo di sistematizzazione e di sintesi, «Filologija» 11, pgs. 329-360.

1984:

86. Le funzioni pragmalinguistiche dei croatismi nei testi rovignesi contemporanei, «Linguistica» 24, pgs. 335-353.

87. L'istroromanzo di fronte alla Romània Perduta tra il friulano ed il romeno, en: D. Messner (ed.) Das Romanische in den Ostalpen, Wien, pgs. 95-110.

88. Kontrastivne bilješke o semantičkim, pragmatičkim i sintaktičkim aspektima izrazâ za restrikciju u hrvatskom ili srpskom i talijanskom jeziku, «StrJez» $1-2$, pgs. 4-20.

89. Latinizmi u pisanim istroromanskim izvorima, «Filologija» 12, pgs. 105-121.

90. O negaciji u jeziku, Zbornik Pedagoškog fakulteta u Rijeci 6, pgs. 189-196.

91a. La formazione delle parole nell'istroromanzo rovignese contemporaneo (I), «L'Italia Dialettale» (ID) 47 (n.s. 24), pgs. 111-182.

1985:

91b. La formazione delle parole nell'istroromanzo rovignese contemporaneo (II), «ID» 48 (n.s. 25), pgs. 35-133.

92. Problemi, esperienze, prospettive nelle ricerche di linguistica istroromanza, en: Homenaje a Álvaro Galmés de Fuentes I, Madrid-Oviedo, pgs. 299-315.

93. 1835-1983: Un secolo e mezzo di tradizione scritta dell'istroromanzo, en: Actes du XVIIème Congrès International de Linguistique et Philologie Romanes 2, Marseille, pgs. 161-178.

94. S'agit-il vraiment de contaminations?, en: Zbornik u čast Petru Skoku, Zagreb, pgs. 483-489.

95. Per un quadro completo dell'analogia nell'evoluzione formale del verbo italiano, en: SLI 23: Linguistica storica e cambiamento linguistico, Roma, pgs. 175-192.

96. Gli idiomi retoromanzi negli "Elementi di linguistica romanza" di Petar Skok, «Ladinia» 9, pgs. 47-54.

1986:

97. Lessicografia istroromanza ieri, oggi, domani, «RLiR» 50, pgs. 5-35.

98. O prevođenju “neprevedivog “( (I) «StrJez» 2, pgs. 74-81; (II) «StrJez» 3-4, pgs. $121-127$.

99. Un'intuizione importante: "Qualche...elaborazione del latino...forse...propria e indigena dell'Istria", en: G.I. Ascoli, Attualità del suo pensiero a 150 anni dalla nascita, Firenze, pgs. 149-158. 
100. La formazione delle parole nella "Descrizione dei mesi" di Josèf Butačon, en: Raetia Antiqua et Moderna, W.Th. Elwert zum 80. Geburtstag, Tübingen, pgs. 481-498.

101. L'elemento tedesco nel Rovignese contemporaneo dell'antologia Istria Nobilissima, «Vox Romanica» 45, pgs. 13-25.

102. Neologismi tecnici ed affini nella prosa rovignese attuale, «Linguistica» 26, pgs. $69-82$.

1987:

103. Frasi dipendenti performative nell'istroromanzo odierno, en: Romania et Slavia Adriatica, Festschrift für Žarko Muljačić, Hamburg, pgs. 373-388.

104. Le convergenze e le divergenze fonetiche nell'istroromanzo (soprattutto rovignese) ed i loro riflessi nei test $i$, «RLiR» 51, pgs. 331-350.

105. Historijska tumačenja u učenju jezika, «StrJez» 2, pgs. 75-86.

106. Stratificazione di contatti e concordanze e differenziazione linguistica, «Mediterranean Language Review» 3, pgs. 33-54.

107. Toponomastika u suvremenom studiju retoromanskih govora, «OnJug» 12, pgs. 1-15.

108. Frasi interrogative nell'istroromanzo rovignese odierno, «Linguistica» 27, pgs. 73-85.

109. Le frasi dipendenti del rovignese attuale, «TraLiLi» 25/1, pgs. 149-179.

110. O latinsko-talijanskim interferencijama u učenju jezika, «Latina et Graeca», 30, Zagreb, pgs. 17-24.

1988:

111. I caratteri principali della sintassi del rovignese scritto attuale, «RLiR» 52 , pgs. 35-53.

112. Onomastički elementi u suvremenoj rovinjskoj književnosti, "Čakavska rič» 1 , Split, pgs. 91-100.

113. Sintassi, semantica, pragmatica in alcuni interessanti casi di ambiguità nei testi rovignesi moderni, «Linguistica» 28 , pgs. 67-74.

114. L'istroromanzo in una recente pubblicazione linguistica. Aggiunte, commenti, rettifiche, risposte alla problematica istroromanza nel volume omaggio a Žarko Muljačić Romania et Slavia Adriatica, ib., pgs. 111-124.

115. O jednom tipu dvočlanih složenica u hrvatskom ili srpskom i talijanskom je$z i k u$, «Filologija» 16 , pgs. 145-150.

1989:

116. Nešto o izgovoru latinskog jezika (ovaj put s romanističkog gledišta), «Marulić», Zagreb, 4, pgs. 429-435.

117. Prema kontrastivnoj pragmatici tzv. "čestica" u hrvatskom ili srpskom i talijanskom jeziku, «Rad» JAZU 427, pgs. 127-194.

118. Izražavanje intenziteta svojstva u hrvatskom ili srpskom $i$ talijanskom jezi$k u$, «StrJez» 2, pgs. 63-69. 
119. La negazione nel rovignese scritto contemporaneo, «Italica Belgradensia» 2, pgs. 9-26.

120. Le interiezioni (fonosimboli) nella prosa rovignese dell'antologia "Istria Nobilissima “, «Linguistica» 29, pgs. 71-80.

121. Alcune riflessioni a proposito di una recentissima grammatica della lingua italiana, ib., pgs. 149-160.

1990:

122. Aspetti della polimorfia in istroromanzo, en: Studi in memoria di Ernesto Giammarco, Pisa, pgs. 381-387.

123. O talijanskoj leksičkoj komponenti u Lučbi za svakoga Bogoslava Šuleka, «Rasprave Zavoda za jezik» 16, pgs. 275-293.

1991:

124. Imena u najnovijem rječniku jednoga istroromanskog govora, «OnJug»14, pgs. $133-139$.

125. Neka objašnjenja o latinsko-romanskom kontinuitetu $i$ statusu latinskog jezika danas, «Marulić» 1, pgs. 87-93.

C. Comptes rendus, points de vue, notules

1962:

126. Bertil Malmberg: André Martinet, Économie des changements phonétiques, «Studia Neophilologica» $31 / 2$ (1959); «SL»1, pgs. 1-4.

1963:

127. V. Vinja, Gramatika španjolskog jezika s osnovama španjolsko-francuskotalijanskog uspoređenja, «SRAZ»15-16, pgs. 237-241.

1964:

128. Fonološke studije Haralda Weinricha, «SL» 3, pgs. 125-134.

1967:

129. M. Savić - S. Musić - S. Šlenc, Staroitalijanski tekstovi (XIII i početak XIV veka), «SRAZ» 23, pgs. 224-229.

130. Roger L. Hadlich, The Phonological History of Vegliote, «SL» 4, pgs. $167-185$.

\section{8:}

131. V. Vinja, Elément grec dans la phytonymie serbocroate de l'aire dalmate, «SRAZ» 25-26, pg. 186 (notule).

132. Ž. Muljačić, Distinktivna obilježja latinskih fonema, ib., pg. 186 (notule). 
133. A. Kovačec, Observations sur les influences croates dans la grammaire istroroumaine, ib., pgs. 187-188 (notule).

1970:

134. «Lingua e stile» $I-I I I$, «Filologija» 6, pgs. 378-382.

135. P.A. Gaeng, An Inquiry into Local Variations in Vulgar Latin as Reflected in the Vocalism of Christian Inscriptions, «RRL»15/4, pgs. 404-411.

1971:

136. R. Flora, Rumunsko-srpskohrvatski rečnik (Dicționar român-sîrbocroat), «SRAZ» 29-32, pgs. 650-655.

137. Ž. Muljačić, Les phonèmes $/ \hat{c} /$ et $/ \hat{g} /$ dans les emprunts italiens du serbocroate: problème de transcription et de distance phonématique, ib., pgs. 657-658 (notule).

138. Z̆. Muljačić, Tipologija jezičnog kalka, ib., pg. 658 (notule).

139. Ž. Muljačić, Die slavisch-romanische Symbiose. in Dalmatien in struktureller Sicht, ib., pgs. 658-659 (notule).

1973:

140. A. Kovačec, Descrierea istroromânei actuale, «Filologija» 7, pgs. 274-276.

1974:

141. A. Kovačec, Descrierea istroromânei actuale, «SRAZ» 37, pgs. 295-301.

142. M. Illiescu, Le frioulan à partir des dialectes parlés en Roumanie, «Zeitschrift für romanische Philologie» 90/1-4, pgs. 406-415.

143. S. Kiss, Les transformations de la structure syllabique en latin tardif, «IncLing» 1 , pgs. 165-187.

1975:

144. S. Musić, Romanizmi u severozapadnoj Boki Kotorskoj, «SRAZ» 39, pgs. 235-251.

145. Scritti e ricerche di grammatica italiana, ib., pgs. $251-258$.

146. W. Mair, Ennebergische Morphologie, «IncLing» 2, pgs. 205-220.

1978:

147. Due recenti contributi statunitensi alla linguistica romanza e latina, «Linguistica» 17 , pgs. $161-206$.

1981:

148. «Ladinia» $I-I V$, «Linguistica» 21 , pgs. $325-331$. 
1982:

149. M. Iliescu - V. Neagu - C. Nedelcu - G. Scurtu, Vocabularul minimal al limbii române cu traducere în limbii engleză, franceză, spaniolă pentru studenti străini, «RLiR» 46, pgs. 182-185.

150. E. Pulgram, Italic, Latin, Italian, «Linguistica» 22, pgs. 277-297.

1983:

151. I. Loi Corvetto, L'italiano regionale di Sardegna, «Linguistica» 23, pgs. 313-326.

152. Fonologia etrusca fonetica toscana, ib., pgs. 327-337.

153. «Quaderni dell'Atlante Linguistico Toscano» 1, ib., pgs. 339-347.

154. K.E. Karlsson, Syntax and Affixation, ib., pgs. 349-358.

155. E. Azaretti, Evoluzione dei dialetti liguri (2 ème éd.), «IncLing» 8, pgs. 159-164.

156. Profilo dei dialetti italiani, a cura di Manlio Cortelazzo, «Filologija» 11, pgs. $425-438$.

1985:

157. M. Fogarasi, Parole e cultura giuridica e filosofica, «IncLing»10, pgs. $159-165$.

1986:

158. A. Elia, Le verbe italien, «Linguistica» 26, pgs. 193-197.

159. P. Swiggers, Les conceptions linguistiques des Encyclopédistes, ib., pgs. 198-201.

160. «Ladinia» $V-V I I I$, ib., pgs. 202-210.

161. «Estudis Gramaticals» 1, ib., pgs. 211-215.

162. Novi lingvistički časopis: «SOL - Lingvistički časopis», godina I, broj 1, ib., pgs. 216-218.

162. F. Bruni, L'italiano. Elementi di storia della lingua e della cultura, «Studi Mediolatini e Volgari» (SMV) 32, pgs. 153-160.

164. "Quaderni dell'Atlante Linguistico Toscano» 2/3, ib., pgs. 160-164.

165. W. Pecoraro - Ch. Pisacane, L'avverbio, ib., pgs. 165-168.

1987:

166. M. Dardano - P. Trifone, La lingua italiana, «StrJez» 2, pgs. 124-127.

167. P. Simunović, Istočnojadranska toponimija, «C̆akavska rič» 15/1, pgs. 119-123.

168. V. Vinja, Jadranska fauna, «Linguistica» 27, pgs. 167-174.

169. Studi ladini in onore di Luigi Heilmann, ib., pgs. 175-179.

170. «Ladinia» $I X-X$, ib., pgs. $180-185$.

171. V. Väänänen, Le journal-épître d 'Égérie, «SMV».33, pgs. 279-283. 
1988:

172. M. Fogarasi, Nuovo manuale di storia della lingua italiana, « $\mathrm{RLiR} » 52$, pgs. $268-275$.

173. Akten der Theodor Gartner-Tagung, «Linguistica» 28, pgs. 153-159.

174. H. Geckeler - D. Kattenbusch, Einführung in die italienische Sprachwissenschaft, ib., pgs. 160-163.

175. III Rëscontr Antërnassional dë Studi an sla Lenga e la Literatura Piemontèisa, Alba 1986 [1987]; IV Rëscontr ecc. Alba 1987 [1988], «IncLing» 12, pgs. 179-183.

176. «SOL» 2-4, «Zbornik Matice Srpske za filologiju i lingvistiku» (ZMSFL) $31 / 1$, pgs. $193-199$.

1989:

177. L. Heilmann, Linguaggio, lingue, culture, «Mediterranean Language Review» 4-5, pgs. $172-178$.

178. C. Ciseri Montemagno, Dai linguaggi alla cultura. Introduzione all'educazione linguistica nella scuola elementare, «Linguistica» 29, pgs. $169-172$.

179. E. Blasco Ferrer, Le parlate dell'Alta Ogliastra, ib., pgs. 173-178.

180. Vocabularul reprezentativ al limbilor romanice, ib., pgs. 179-185.

181. «SOL» 5-7, «ZMSFL» 32/2, pgs. 229-234.

182. P. Agazzi - A. Fallica - A. Menegoi, La lingua e i testi, «StrJez» 4, pgs. 206-210.

183. Beiträge zu Sprachvergleich und Sprachentwicklung, Festschrift zum 60. Geburtstag von Gustav Ineichen, «SMV» 35, pgs. 288-293.

1990:

184. Il romanesco ieri e oggi, «RLiR» 54 , pgs. 235-238.

185. M. Sala, El problema de las lenguas en contacto, ib., pgs. 269-273.

186. M. Cortelazzo, Venezia, il Levante e il mare, ib., pgs. 519-523.

187. Enciclopedia limbilor romanice, ib., pgs. 529-538.

188. V Rëscontr Antërnassional dë Studi an sla Lenga e la Literatura Piemontèisa, Alba 1988 [1989], ib., pgs. 574-576.

189. G.B. Mancarella, Ricerche linguistiche a Tursi, ib., pgs. 576-579.

190. A. Harder, Laut- und Formenlehre der Mundart von Ripatransone, «Linguistica» 30, pgs. 207-210.

191. H. Siller-Runggaldier, Grödnerische Wortbildung, ib., pgs. 211-217.

192. F. Rainer, I nomi di qualità nell'italiano contemporaneo, ib., pgs. 218-223.

193. «Ladinia» $X I-X I I$, ib., pgs. $224-228$.

1991:

194. VI Rëscontr Antërnassional dë Studi an sla Lenga e la Literatura Piemontèisa, Alba 1989 [1990], «RLiR» 55, pgs. 215-217. 
195. Lars Larsson, La sintassi dei pronomi relativi in italiano moderno ecc., Uppsala 1990 , ib., pgs. 233-237.

D. Varia

1961:

196. Strani pisci, Ière éd., Zagreb: 19 articles sur des écrivains portugais, brésiliens et roumains.

1968:

197. Strani pisci, IIème éd., Zagreb: 12 articles sur des écrivains roumains.

1978:

198. Presentazione en: G.A. Dalla Zonca, Vocabolario dignanese-italiano, a cura di Miho Debeljuh, Trieste, pgs. VII-X.

\section{3:}

199. O jeziku Zaninijevih pjesama (Rovinjski i drugi istroromanski dijalekti), en: Ligio Zanini, Razgovor $s$ galebom Filipom, "Istra kroz stoljeća", kolo IV, knjiga 24, Pula - Rijeka, pgs. 303-307.

1988:

200. Istroromanski dijalekti, Enciklopedija Jugoslavije 5, Zagreb, pgs. $652^{\mathrm{b}}$ $653^{\mathrm{b}}$.

1989:

201. Réponses à "Pet pitanja profesoru Pavlu Tekavčiću", SOL 8, pgs. 1-6.

202. Commentaire à l' Articolo commentato/Commented article de M.Alinei Il problema della datazione in linguistica storica, "Quaderni di semantica» XII, num. 1, pgs. 35-37.

\section{E. Les travaux sous presse}

203. Riflessi di idiomi, popoli, culture nella lingua della prosa rovignese contemporanea, en «BALM», nro in memoriam de Mirko Deanović.

204. Aspetti linguistici di una traduzione rovignese dell'Episodio del Conte Ugolino, en Hommage Rupprecht Rohr, Mannheim.

205. Sul condizionale in istroromanzo, en Hommage Maria Iliescu, Innsbruck.

206. Sull'affettività nei dialetti istroromanzi, en Hommage à Giuseppe Francescato, Trieste.

207. Onomastika u rumunjskoj »Enciklopediji romanskih jezika«, en «OnJug». 
208. Gli idiomi retoromanzi nell'Enciclopedia limbilor romanice, en «Ladinia».

209. Pragmatika i lingvistika teksta u studiju jezika suvremene rovinjske istroromanske književnosti, en «Dometi», Rijeka.

210. G.A. Dalla Zonca (en collaboration avec prof. Miho Debeljuh), dans le IIIème volume de Hrvatski biografski leksikon.

Bibliographie arrêtée au 30 Septembre 1991. Rédigée par M. Tekavčić. 